\title{
Optimal imaging of infiltrating lipomas of the neck
}

\begin{abstract}
Infiltrating lipomas are rare benign, slow-growing tumours. They characteristically infiltrate skeletal muscle and have a propensity for local recurrence if incompletely resected. We present a patient in whom preoperative magnetic resonance imaging (MRI) was predictive of an infiltrating lipoma of the neck. Complete resection of the lipoma was undertaken leading to resolution of symptoms. There was no evidence of recurrence three years postoperatively. The scanning protocol, disease extent, predictive radiological features, and advantages of MRI over other imaging modalities for this tumour are discussed.
\end{abstract}

Keywords: lipoma, infiltrating, magnetic resonance imaging
Volume 3 Issue 3 - 2015

\author{
Mary Louise Montague, Musheer Hussain SS \\ Department of Otolaryngology, The Royal Hospital for Sick \\ Children, UK
} Correspondence: Mary Louise Montague, Department
Otolaryngologist, The Royal Hospital for Sick Children, 9 Sciences Road, Edinburgh, EH2I 7TU, UK, Tel 0131 5360830 , Email mary-louise.montage@luht.nhs.uk

Received: April 12, 20I5 | Published: September 2I, 20I5

\section{Introduction}

Lipomas have been reported to occur at sites throughout the body. Only $13 \%$ occur in the head and neck region. Of these the majority are found in the subcutaneous tissues of the neck. ${ }^{1}$ Such lesions may, depending upon their position and extent, cause significant symptoms. On occasion they may be deep-seated and infiltrate between or within muscles. It is possible to differentiate between circumscribed lipomas and infiltrating lipomas radiologically. We present a patient in whom a pre-operative MRI scan was predictive of an infiltrating lipoma of the neck.

\section{Case report}

A 68-year old Caucasian female presented to the Otolaryngology clinic for evaluation of hoarseness. Fibreoptic laryngoscopy revealed partial left vocal cord palsy. A lobulated cystic mass in the left side of the neck was noted on neck palpation. The patient reported this to be asymptomatic and to have been present from infancy with gradual enlargement. A chest radiograph was normal. Computed tomography of the neck and thorax demonstrated a large radiolucent mass in the left neck consistent with the diagnosis of a lipoma. This extended from the mastoid tip cranially to the body of the fourth cervical vertebra caudally (Figure 1). No laryngeal or intrathoracic abnormality was found and the vocal cord palsy was considered to be idiopathic. Further examination after a course of voice therapy confirmed normal symmetrical vocal cord movement with restoration of voice.

The patient returned to the Otolaryngology clinic seven years later reporting an increase in the size of the left-sided neck mass with symptoms of progressive local discomfort and dyspnoea. Clinical examination revealed an $8 \mathrm{~cm}$ by $6 \mathrm{~cm}$ firm multi-lobulated swelling extending anteriorly across the mandible (Figure 2). There was no evidence of airway compromise on physical examination.

A contrast enhanced magnetic resonance imaging (MRI) scan demonstrated a well-defined homogeneous mass lesion in the left side of the neck (Figure 3). Signal characteristics were consistent with a lipoma. It appeared to have arisen in the fat space deep to the left sternomastoid muscle which was significantly enlarged. The left carotid sheath was displaced medially into the parapharyngeal space.

Complete elective transcervical resection was performed. Infiltration of the parapharyngeal space, anterior and posterior triangles of the neck, pterygopalatine fossa and retropharyngeal space was confirmed intraoperatively. The mass was resected en bloc and macroscopically comprised a $9 \times 6.5$ x $4 \mathrm{~cm}$ lobulated incompletely encapsulated fibrofatty tumour. Microscopically this comprised sheets of mature adipose tissue with some intervening bands of fibrous connective tissue - entirely consistent with the radiological diagnosis of an infiltrating lipoma. There was no clinical evidence of recurrence three years after resection.

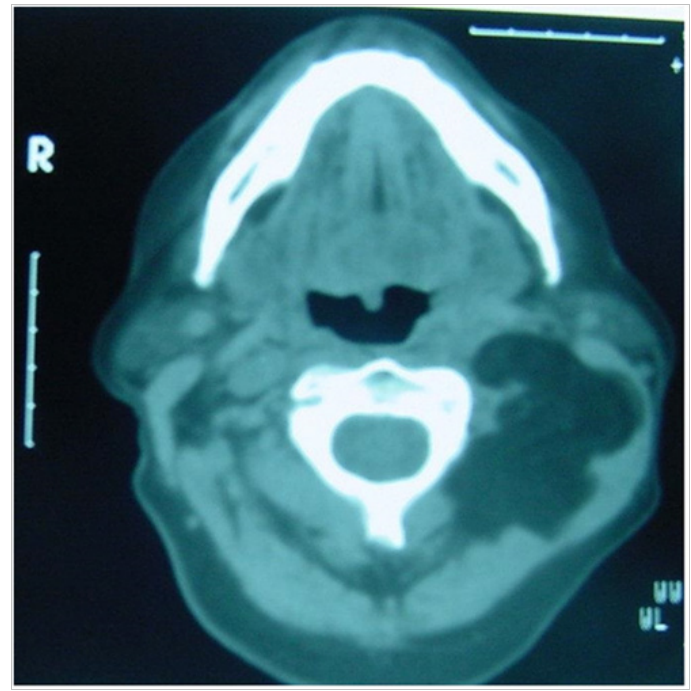

Figure I Computed tomography (axial cut) appearance at initial presentation.

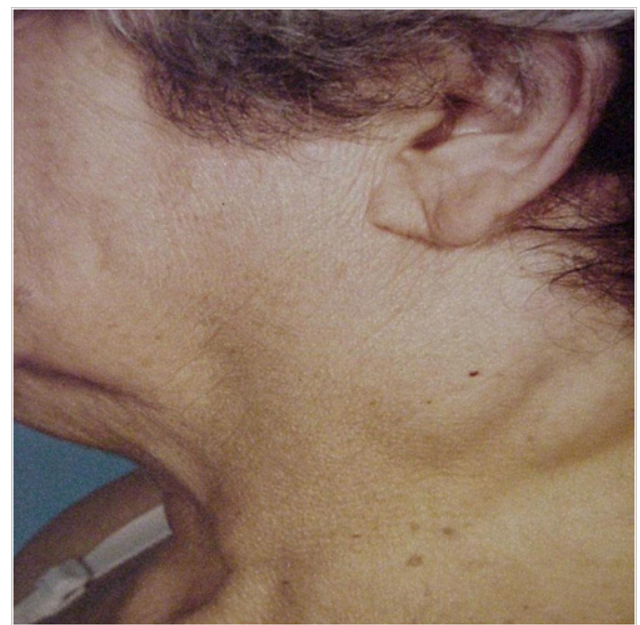

Figure 2 Clinical appearance of left neck swelling seven years after initial presentation. 


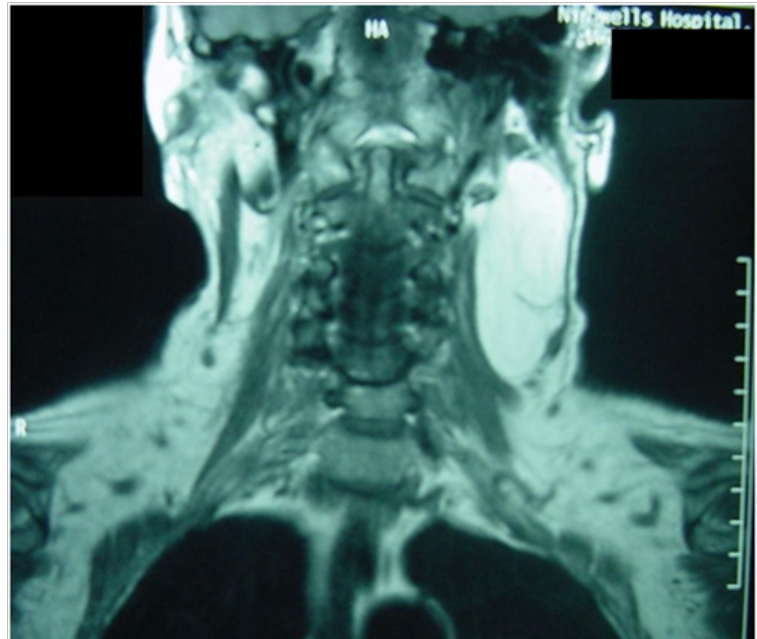

Figure 3 Extent of infiltrating lipoma demonstrated on contrast MRI scan (coronal cut).

\section{Discussion}

Simple lipomas are slow-growing tumours of mesenchymal origin. They are well circumscribed by a delicate fibrous capsule and do not infiltrate. They comprise mature adipose tissue cells, are single or lobulated, and may be soft, firm, or cystic. They have a low rate of recurrence after complete resection. ${ }^{2}$ Infiltrating lipomas are incompletely encapsulated and demonstrate a high rate of recurrence following resection. ${ }^{3}$ They may enlarge rapidly and infiltrate local tissues.

A recurrence rate as high as $62.5 \%$ has been reported for the infiltrating lipoma compared with a 5\% local recurrence rate for an incompletely resented simple lipoma. ${ }^{2}$ The ability of modern imaging techniques to detect infiltration preoperatively can significantly reduce the rate of recurrence by permitting the Otolaryngologist to plan wide local resection of the lipoma.

In the case described there was no histological evidence of skeletal muscle infiltration. But in fact intramuscular infiltration is rarely reported in the head and neck. Most infiltrating lipomas of the neck arise in the intermuscular fascial septae and secondarily infiltrate the muscle. ${ }^{4}$

It is difficult to determine the exact origin of the lipoma described here given its extent depicted radiologically and confirmed intraoperatively. Specifically the infrahyoid portion of the retropharyngeal space and the parapharyngeal space were infiltrated by lipoma. Extension was also demonstrated into the anterior triangle of the neck to involve the submandibular space, into the posterior triangle of the neck behind the carotid sheath and into the pterygopalatine fossa. The tumour may have originated at any one of these sites. The retropharyngeal lipoma is an extremely rare tumour. Only 31 cases are described in the literature. Only two cases of an infiltrating retropharyngeal lipoma have previously been reported. ${ }^{5,6}$ Multiple neighbouring spaces border the retropharyngeal space maintaining a constant relationship with it throughout the extracranial head and neck and providing several routes for extension of an infiltrating lipoma as described here. A plain lateral neck radiograph was not obtained in this case though X-rays demonstrate a radiolucent area in the retropharyngeal space, absence of bone destruction and help detect upper airway compromise.
Ultrasonography, despite being a frequently requested first modality in the assessment of cervical swellings, was not employed in this case either. The diagnosis had been suspected clinically and evaluation of tumour extent and depth was required. Nonetheless cervical lipomas do have a fairly typical morphological appearance on ultrasound. It is not however as pathognomonic as the density values are by means of $\mathrm{CT}^{7}{ }^{7}$ Whilst ultrasound can differentiate between circumscribed lipomas and infiltrating lipomas unfortunately it does not permit an adequate preoperative assessment of depth of infiltration in the presence of an infiltrating lipoma of the neck. Only the cervical vessels can be clearly delineated in this condition.

A CT scan with contrast at initial presentation showed a lowattenuation mass with Hounsfield units of - 43.0 in the left side of the neck extending from the skull base to the level of the fourth cervical vertebra. The characteristic CT appearance of lipoma is that of low attenuation related to fatty tissue (Hounsfield values -50 to -150$)$. The MRI appearance was of a mass with a high-intensity signal on T1-weighted images and an intermediate signal on T2weighted sequences. The appearance of lipomas on CT and MRI has been considered diagnostic, although neither of these imaging techniques can distinguish a lipoma from a liposarcoma. ${ }^{8}$ An irregular radiographic interface between lipoma and muscle tissue has previously been predictive of infiltration histologically. ${ }^{9}$

In the case reported the use of CT scanning proved invaluable in the diagnosis of a lipoma at initial presentation. Greater soft tissue definition provided by contrast MRI seven years later when the patient presented with enlargement of the neck mass permitted better delineation of tumour extent. Specifically it allowed refinement of the original diagnosis to an infiltrating lipoma of the neck.

\section{Conclusion}

It is generally accepted that both CT and MRI can differentiate between simple well-circumscribed lipomas and infiltrating lipomas. We would advocate on the basis of this report that MRI is the optimal imaging modality for infiltrating lipomas occurring in the neck. It should also be the preferred modality for imaging the neck to detect recurrence.

\section{Acknowledgments}

None.

\section{Conflicts of interest}

Author declares there are no conflicts of interest.

\section{Funding}

None.

\section{References}

1. Kakani RS, Bahadur S, Kumar S, et al.Parapharyngeal lipoma. J Laryngol Otol. 1992;106(3):279-281.

2. Benign lipomatous tumours. In: Louis St \& Mosby CV (Eds.), Soft Tissue Tumours. p. 199-2413.

3. Dionne GP, Seemayer TA. Infiltrating lipomas and angiolipomas revisited. Cancer. 1974;33(3):732-738.

4. Austin RM, Mack GR, Townsend CM, et al.Infiltrating (intramuscular) lipomas and angiolipomas. Arch Surg. 1980;115(3):281-284.

5. Eisele DW, Landis GH. Retropharyngeal infiltrating lipoma-a case report. Head Neck Surg. 1988;10(6):416-421. 
6. Senchenkov A, Werning JW, Staren ED. Radiographic assessment of the infiltrating retropharyngeal lipoma. Otolaryngol Head Neck Surg. 2001;125(6):658-660.

7. Gritzmann N, Schratter M, Traxler M, et al.Sonography and computed tomography in deep cervical lipomas and lipomatosis of the neck. $J$ Ultrasound Med. 1988;7(8):455-456.
8. Rosell A, Garcia-Arranz G, Llavero MT, et al.Imaging case study of the month: Lipoma of the retropharyngeal space. Ann Otol Rhinol Laryngol. 1998;107(8):726-728.

9. Scherl MP, Som PM, Biller HF, et al.Recurrent infiltrating lipoma of the head and neck. Arch Otolaryngol Head Neck Surg. 1986;112(11):1210-1212. 